\title{
Cross-species validation of cell cycle arrest markers for acute kidney injury in the rat during sepsis
}

Zhi-Yong Peng ${ }^{1,2+}$, Feihu Zhou ${ }^{3 \dagger}$ and John A. Kellum ${ }^{1 *}$

\author{
* Correspondence: \\ Kellumja@upmc.edu \\ ${ }^{\dagger}$ Equal contributors \\ ${ }^{1}$ Department of Critical Care \\ Medicine, The Center for Critical \\ Care Nephrology, CRISMA (Clinical \\ Research, Investigation, and Systems \\ Modeling of Acute IIIness) Center, \\ University of Pittsburgh School of \\ Medicine, 604 Scaife Hall, 3550 \\ Terrace Street, Pittsburgh, PA 15261, \\ USA \\ Full list of author information is \\ available at the end of the article
}

\begin{abstract}
Background: The recent discovery of cell cycle arrest biomarkers, tissue inhibitor of metalloproteinases (TIMP)-2 and insulin-like growth factor binding protein 7 (IGFBP7), has led to a newly available clinical test for acute kidney injury. The performance of these markers in preclinical studies has not been established. Therefore, we sought to evaluate the performance of TIMP-2 and IGFBP7 in rats undergoing cecal ligation and puncture.
\end{abstract}

Methods: In this secondary analysis, we analyzed banked urine samples from 60 Sprague-Dawley rats undergoing cecal ligation and puncture (CLP). Samples were obtained from baseline, $18 \mathrm{~h}$ after CLP, at the end of fluid resuscitation (22 h after CLP), and again $24 \mathrm{~h}$ later. We measured TIMP-2 and IGFBP7 and compared the results to acute kidney injury by RIFLE criteria for creatinine using area under the receiver operating characteristic curve (AUC). The primary endpoint was moderate-to-severe acute kidney injury (AKI) (I or F criteria), and the primary time point was immediately after fluid resuscitation. Secondary outcomes included mortality and comparisons with other biomarkers: cystatin $C$ and neutrophil gelatinase-associated lipocalin (NGAL) in both urine and plasma.

Results: After fluid resuscitation, urine [TIMP-2] and [IGFBP7] were significantly higher in animals developing moderate-to-severe AKI $(p=0.002$ and $p=0.01)$. AUC of [TIMP-2].[IGFBP7] for AKI was 0.89 (95\% Cl 0.80-0.98). By contrast, the next best AUC was seen with plasma cystatin C $(0.78 ; 95 \% \mathrm{Cl} \mathrm{0.65-0.90)}$. [TIMP-2].[IGFBP7] also predicted mortality (AUC 0.69; $95 \%$ Cl 0.53-0.85).

Conclusions: In this experimental model of sepsis in the rat, cell cycle arrest biomarkers TIMP-2 and IGFBP7 are valid predictors of acute kidney injury.

Keywords: Acute kidney injury, Sepsis, Tissue inhibitor of metalloproteinases (TIMP)-2, Insulin-like growth factor binding protein 7 (IGFBP7), Cell cycle arrest, Biomarkers

\section{Background}

Recently, two novel urinary biomarkers, tissue inhibitor of metalloproteinases-2 (TIMP-2) and insulin-like growth factor-binding protein 7 (IGFBP7), were validated for predicting moderate-to-severe acute kidney injury (AKI) (stages 2 and 3 by Kidney Disease Improving Global Outcome (KDIGO) [1] criteria) in critically ill patients [2, 3]. Both TIMP-2 and IGFBP7 are markers of G1 cell-cycle arrest, which prevents cells from dividing when

(C) 2016 Peng et al. Open Access This article is distributed under the terms of the Creative Commons Attribution 4.0 International License (http://creativecommons.org/licenses/by/4.0/), which permits unrestricted use, distribution, and reproduction in any medium, provided you give appropriate credit to the original author(s) and the source, provide a link to the Creative Commons license, and indicate if changes were made. 
potentially injured [4], and both appear to respond to a wide variety of cellular stressors including inflammation, ischemia, oxidative stress, drugs, and toxins [5-7]. Furthermore, both molecules also act as "alarm" proteins exerting paracrine effects on adjacent cells [8]. Novel AKI biomarkers may play important roles clinically [9], but they also have great potential to transform clinical trials $[10,11]$. As such, it is important to understand how these markers perform in preclinical models of AKI. Sepsis is the most common cause of AKI in critically ill patients [12, 13], and cecal ligation and puncture (CLP) in small animals is a commonly used preclinical model.

Thus, we sought to evaluate the performance of [TIMP-2].[IGFBP7] in an rat model of sepsis. In order to be consistent with the human trials that were used to discover and validate these biomarkers [2,3], we used moderate-to-severe AKI (equivalent to KDIGO stages $2-3$ or RIFLE I-F) as the primary outcome. We assessed biomarker performance at various time points, but we used the immediate post-resuscitation time point as primary because it most closely matches the time when patients were enrolled in these trials.

\section{Methods}

For this analysis, we used banked samples from 60 animals used in a prior laboratory experiment involving rats subject to cecal ligation and puncture and randomized to resuscitation with two different crystalloids: $0.9 \%$ saline and plasmalyte. Complete details of the parent study have been published [14]. Briefly, 24- to 28-week-old (weight 400-600 g) male, Sprague-Dawley rats were anesthetized with intraperitoneal injection of pentobarbital sodium $(50 \mathrm{mg} / \mathrm{kg}$ ) and subjected to CLP with a predetermined $25 \%$ ligated length of cecum and 18-gauge needle: two punctures inferior to the ileocecal valve. Eighteen hours after CLP animals were re-anesthetized, vascular cannula were placed and animals received $10 \mathrm{ml} / \mathrm{kg}$ fluid resuscitation in the first hour with either $0.9 \%$ saline or plasmalyte. For the next $3 \mathrm{~h}$, animals received the same fluid at $5 \mathrm{ml} / \mathrm{kg} / \mathrm{h}$. Animals were observed for survival over an additional $24 \mathrm{~h}$. Blood $(1 \mathrm{ml})$ was drawn from the arterial line, and urine (1-2 ml) was taken from the bladder at 0 (baseline), 18 (before fluid resuscitation), 22 (end of fluid resuscitation), and $24 \mathrm{~h}$ after fluid resuscitation (46 h after CLP). Isolated plasma and urine was kept at $-80{ }^{\circ} \mathrm{C}$ for subsequent neutrophil gelatinase-associated lipocalin (NGAL), cystatin $\mathrm{C}$, and creatinine $(\mathrm{Cr})$ measurements and the remainder stored.

Plasma NGAL and urine NGAL were determined using ELISA (BioPorto Diagnostics, Gentofte, Denmark). Plasma creatinine was detected with a creatinine enzymatic assay kit (BioVision Technologies, Mountain View, CA). Plasma cystatin C and urine cystatin $\mathrm{C}$ were measured by ELISA (BioVendor LLC, Candler, NC). Frozen urine samples were used for measurement of TIMP-2 and IGFBP7 by ELISA. The ELISA for TIMP-2 was made using capture and detect antibodies from R\&D Systems (Minneapolis, MN) and the ELISA for IGFBP7 using capture and detect antibodies from Thermo Fisher Scientific (Waltham, MA) and R\&D Systems, respectively. TIMP-2 and IGFBP7 protein from Abcam (Cambridge, UK) and U-Protein Express (Utrecht, Netherlands), respectively, were used for calibration. The calibration range for both assays was 0.0156 to $2 \mu \mathrm{g} / \mathrm{mL}$.

We determined the severity of AKI using the serum creatinine portion of the RIFLE criteria [15], which classified risk (R), injury (I), and failure (F), on the basis of maximum creatinine increase of 150,200 , and $300 \%$, respectively, in the 2 days 
following CLP. For the primary analysis, we compared I and F to $\mathrm{R}$ and no AKI. Biomarker data are expressed as means \pm standard error (SE). Student's $t$ tests were applied to compare means. Biomarker concentrations were compared across the first three time points using repeated measures ANOVA with Huynh-Feldt correction. The fourth time point was not included because only 19 rats had both TIMP-2 and IGFBP7 results for all four time points. Dunnett's test was used for pairwise comparisons with the baseline time point as the control. We calculated area under the receiver operator characteristic curve (AUC) and $95 \%$ confidence intervals [16]. Analyse-it (Analyse-it Software, Ltd., UK) and XLSTAT (Addinsoft, Paris, France) software were used for statistical analysis. A two-sided $P<0.05$ was considered statistically significant.

\section{Results}

TIMP-2, IGFBP7, and AKI

Samples were available at 18 and $22 \mathrm{~h}$ (right before and after fluid resuscitation) from 48 animals. Of these, 30 developed RIFLE I/F (18 I and 12 F) and 18 either had no AKI (8) or R (10). For animals developing AKI, creatinine peaked most often, at the 48-h time point. Immediately after fluid resuscitation, $22 \mathrm{~h}$ after CLP, urine concentrations of both TIMP-2 and IGFBP7 were greater in animals who ultimately manifested RIFLE I/F compared to those who did not (Fig. 1). The AUC for [TIMP-2].[IGFBP7] for AKI was 0.89 (95\% CI 0.80-0.98). Interestingly, the AUCs for individual markers were significantly $(p<0.05)$ lower than the composite $(0.76$ and 0.72 for TIMP-2 and IGFBP7, respectively). There were no significant differences between the performance of TIMP-2 and IGFBP7 in animals receiving saline or plasmalyte. Mean [TIMP-2].[IGFBP7] at 18 and $22 \mathrm{~h}$ were similar for animals receiving plasmaltye compared to saline (at $18 \mathrm{~h}$

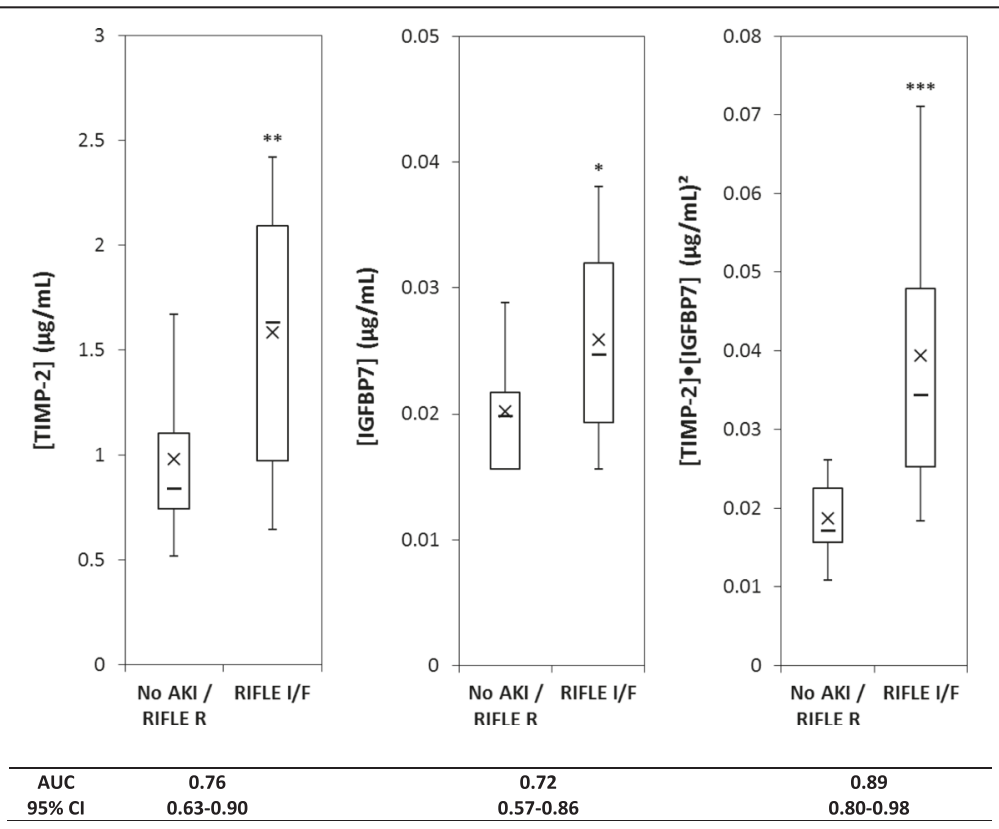

Fig. 1 Urinary [TIMP-2], [IGFPB7], or [TIMP-2].[IGFPB7] levels from animals with no AKI (or only RIFLE R) $(n=18)$ compared to RIFLE I or $F(n=30)$. Box and whiskers show interquartile range and 5 th to 95 th percentiles, respectively. Horizontal dash shows the median, and $X$ shows the mean. ${ }^{*} P=0.01,{ }^{* *} P=0.002,{ }^{* * *} P<0.001$ 
0.034 (95 \% CI $0.023-0.046)$ vs. $0.028(\mu \mathrm{g} / \mathrm{ml})^{2}(95 \%$ CI $0.017-0.039), p=0.43$ or at $22 \mathrm{~h}$ 0.034 (95 \% CI $0.025-0.042)$ vs. $0.029(\mu \mathrm{g} / \mathrm{ml})^{2}$ (95 \% CI 0.022-0.037), $\left.p=0.43\right)$. However, fewer animals developed the endpoint in the plasmalyte group as reported previously [14]. At baseline, [TIMP-2].[IGFBP7] results were not different between those animals who ultimately developed AKI compared to those who did not (Fig. 2). Both TIMP-2 and IGFBP7 increased $18 \mathrm{~h}$ after CLP compared to baseline $(p=0.01$ and $p<0.0001$, respectively). However, neither [TIMP-2].[IGFBP7] (Fig. 2) nor individual markers (data not shown) discriminated well for AKI at the 18-h (pre-resuscitation) time point.

\section{Comparison with other urine biomarkers}

Performances for various AKI biomarkers are shown in Table 1. The same time point (immediately after resuscitation) and same outcome, RIFLE I/F, were used for all comparisons. The AUC for [TIMP-2].[IGFBP7] was significantly greater than for urine NGAL (0.89 vs. $0.71, P<0.05)$ or plasma NGAL (0.89 vs. $0.62, P<0.05$; Table 1$)$.

\section{Mortality}

Early mortality (prior to day 2 after CLP) was observed in 19 animals. All deaths exhibited AKI. Immediately after fluid resuscitation, $22 \mathrm{~h}$ after CLP, urine concentrations of IGFBP7 $(P=0.002)$ but not TIMP-2 $(P=0.48)$ were greater in animals who died prior to day 2 compared to those who did not (Fig. 3). The AUC for [IGFBP7] alone was 0.76 (95\% CI 0.63-0.90) whereas the AUC for [TIMP-2].[IGFBP7] for AKI was 0.69 (95\% CI 0.53-0.85).

\section{Discussion}

Prior animal studies have examined TIMP-2 in relation to AKI [17, 18], and several studies have examined IGF binding proteins in renal disease [19] but none have directly

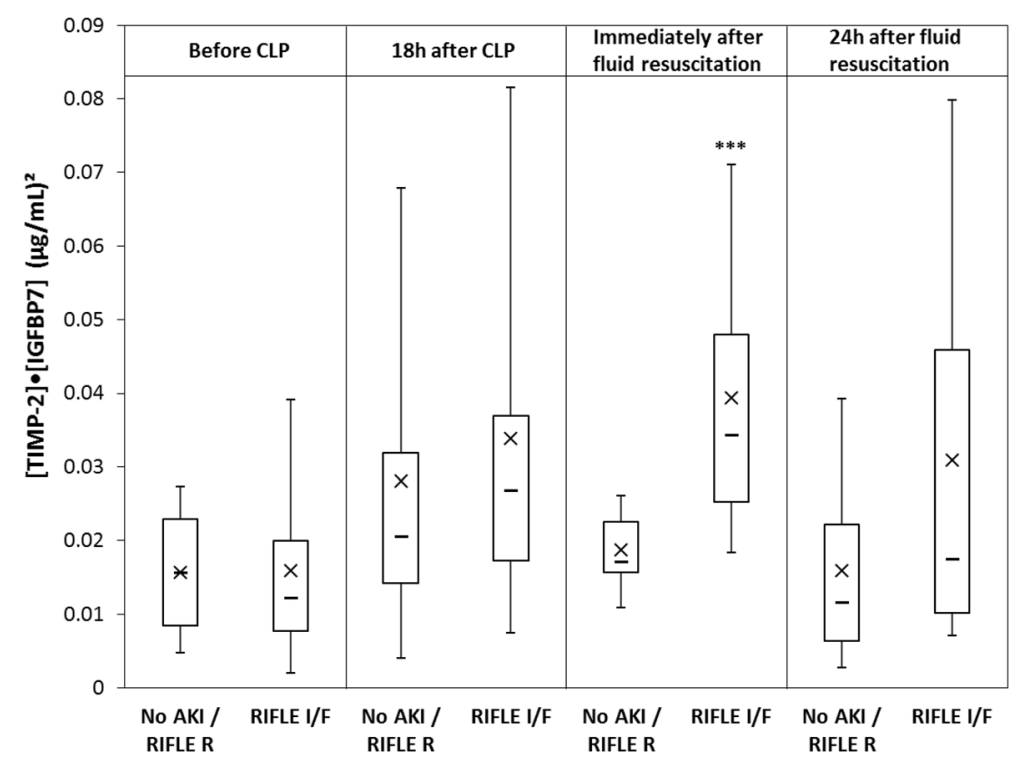

Fig. 2 Box-and-whisker plots for [TIMP-2].[IGFPB7] at various time points comparing no AKI or RIFLE R versus RIFLE I or F. ${ }^{* *} p<0.001$ for immediately after fluid resuscitation 
Table 1 Comparison of biomarkers for predicting RIFLE I/F

\begin{tabular}{lllll}
\hline Biomarker & No AKI & AKl & $p$ value & AUC \\
\hline [TIMP-2]·[IGFBP7] $\left(\mathrm{\mu g}^{2} / \mathrm{mL}^{2}\right)$ & $0.019 \pm 0.001$ & $0.039 \pm 0.003$ & $<0.0001$ & $0.89(0.80-0.98)$ \\
Urine NGAL $(\mathrm{IU} / \mathrm{mL})$ & $2157.6 \pm 102.4$ & $2472.8 \pm 123$ & 0.04 & $0.71(0.63-0.80)$ \\
Plasma NGAL $(\mathrm{IU} / \mathrm{mL})$ & $2143.3 \pm 29.6$ & $2077 \pm 44.5$ & 0.21 & $0.62(0.46-0.79)$ \\
Urine cystatin C $(\mathrm{IU} / \mathrm{mL})$ & $1609.8 \pm 284.6$ & $2572.8 \pm 353.6$ & 0.03 & $0.75(0.56-0.93)$ \\
Plasma cystatin C $(\mathrm{IU} / \mathrm{mL})$ & $1027.3 \pm 185.8$ & $2344.9 \pm 462.5$ & $<0.01$ & $0.78(0.65-0.90)$
\end{tabular}

All comparisons are for the post-resuscitation (primary) time point. Shown are means \pm standard error and areas under the receiver operating characteristic curves (AUC) with $95 \%$ confidence intervals. $P$ values are for two-sided $t$ test

determined the performance of [TIMP-2].[IGFBP7] for the prediction of RIFLE I/F AKI in an experimental model. Thus, to our knowledge, this is the first cross-species validation of [TIMP-2].[IGFBP7] for AKI and establishes the test as a viable tool in the preclinical space. This is important because clinical trials that use [TIMP-2].[IGFBP7] for enrichment will benefit from preclinical data that closely matches the trial design. For this reason, we designed this laboratory investigation to simulate the clinical situation as much as possible. We used moderate-to-severe AKI (RIFLE I/F = KDIGO $2 / 3$ ) as the endpoint, and we tested biomarkers at a time that closely mimics enrolment in clinical trials as well as clinical use in general.

There were several unexpected results of our investigation. First, earlier measurement of [TIMP-2].[IGFBP7] (at $18 \mathrm{~h}$ after CLP and prior to fluids), while demonstrating an increase compared to baseline, was not predictive of RIFLE I/F AKI. We speculate that early increases in TIMP-2 and IGFBP7 might have been protective for some animals while sustained elevations were consistently associated with development of AKI. This result may help explain recent findings in humans where early release of TIMP-2 and IGFBP7 was associated with the protection from AKI in the setting of remote ischemic

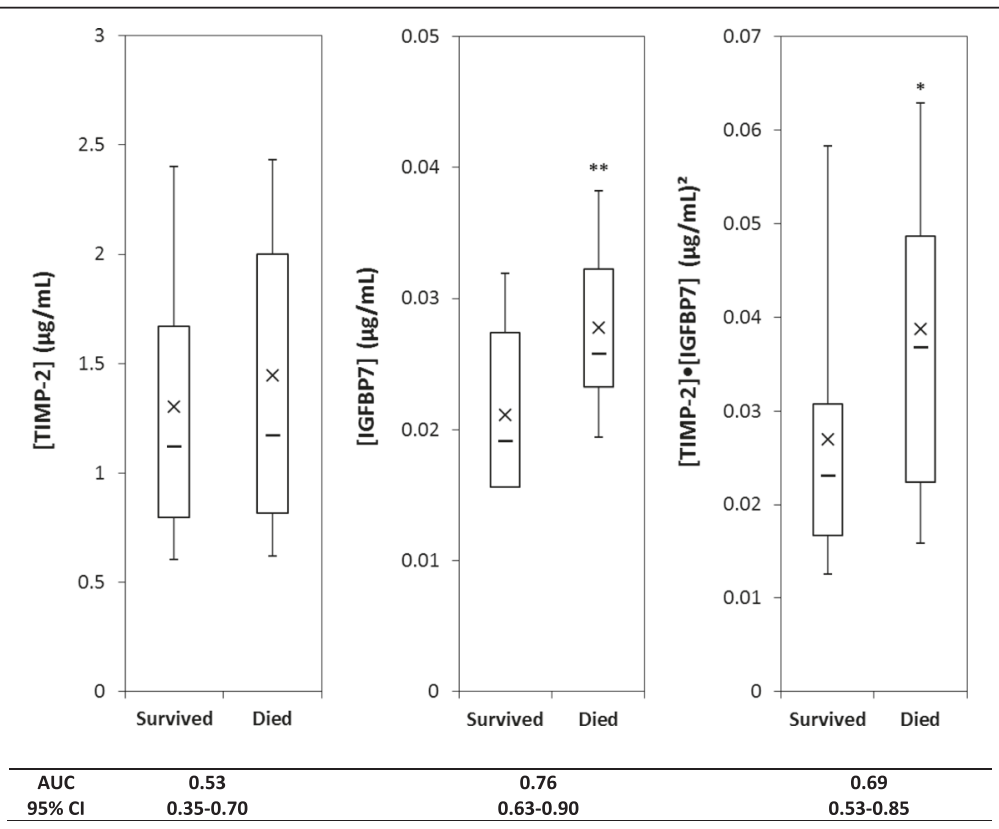

Fig. 3 Urinary [TIMP-2], [IGFPB7], or [TIMP-2].[IGFPB7] levels from animals that survived $(n=29)$ compared to those that died $(n=19)$. Box and whiskers show interquartile range and 5th to 95th percentiles, respectively. Horizontal dash shows the median, and $X$ shows the mean. ${ }^{*} P=0.03,{ }^{*} P=0.002$ 
preconditioning and subsequent cardiac surgery [11]. The mechanisms responsible for this effect are still unclear, but we have proposed that limb ischemia releases damageassociated molecular patterns that signal the kidney to release TIMP-2 and IGFBP7 as part of an "alarm" that protects cells from subsequent injury [20]. Since temporary cell cycle arrest is a protective mechanism, it is reasonable to expect that under certain circumstances the mechanism works and release of TIMP-2 and IGFBP7 is not followed by AKI [9]. We speculate that pre-resuscitation levels may identify "renal stress" but not necessarily predict the effectiveness of the resuscitation. Whereas postresuscitation levels may be more predictive of the pathologic (as opposed to adaptive) state. Alternatively, animals at pre-resuscitation may in fact already have renal injury but it may still be reversible. For animals that responded to resuscitation, they rapidly resolved this injury and no longer exhibited biomarker signatures nor did they manifest clinical AKI. Further study will be necessary to test these hypotheses. However, the finding does have implications for clinical trials and clinical care. For example, the test may have less utility in the pre-hospital arena. Second, it may point to a need to retest patients if clinical evaluation suggests that they were not resuscitated on initial testing.

Another unexpected finding was the wide separation between the AUCs for TIMP-2 and IGFBP7 compared to [TIMP-2].[IGFBP7] for AKI. These differences were much greater than what was observed in humans [2]. Interestingly, in the clinical study by Kashani et al., TIMP-2 had a superior AUC compared to IGFBP7 in patients with sepsis, whereas the opposite was seen in surgical patients [2]. We observed better performance for TIMP-2 in this experimental model of sepsis (Fig. 1). These findings suggest that sepsis-induced AKI may have a unique underlying pathobiology.

Finally, we observed that while TIMP-2 was a better performing marker for AKI, it was a very poor predictor of mortality and IGFBP7 was better for this outcome (AUC 0.76 vs. 0.53$)$; though not quite significant $(p=0.06)$ (Fig. 3). This uncoupling of predictors of AKI and mortality was unexpected and may warrant further investigation.

Our study also showed that urine [TIMP-2].[IGFBP7] was superior to NGAL. NGAL derivation and validation studies were primarily performed in ischemic or nephrotoxic AKI, while investigations of NGAL in septic AKI have demonstrated variable results [21]. NGAL is known to be released by activated neutrophils and appears to be elevated in sepsis, which may complicate its diagnostic value for AKI in patients with sepsis. Our study also used RIFLE I/F AKI criteria (creatinine only) as the primary endpoint so as to more closely model the clinical scenario in humans. Of note, we have previously shown that in antibiotic-treated animals using this exact model, 1-week mortality for RIFLE-F was $36.4 \%$ [22]. This is remarkably similar to $40.3 \%$ crude hospital mortality reported for critically ill patients with KDIGO stage 3 AKI (using creatinine alone) [23] or the $32.4 \%$ covariate-adjusted hospital mortality for KDIGO stage 3 AKI (using both creatinine and urine output) [13].

Our study has important limitations. As a secondary analysis, we were limited in what time points were available. It would have been useful, for example, to have measurements every 1-2 h. However, it is unlikely that we could have obtained this frequency of monitoring in our rats even if we would have tried. Secondly, we chose a relatively high severity model with substantial early mortality ( $40 \%$ by day 2 ). It is not known whether a less severe model would have resulted in similar or different results. Finally, our model involved fluid resuscitation with saline in half of the animals, and 
indeed, the majority of animals reaching the AKI endpoint received saline. Therefore, the exposure is "sepsis plus saline" rather than sepsis alone. However, this mirrors the clinical reality in much of the world especially North America where sepsis patients are invariably resuscitated with saline [24].

\section{Conclusions}

In conclusion, we have validated the [TIMP-2].[IGFBP7] test in an experimental model of sepsis-associated AKI using CLP. Our results establish the test in the preclinical space.

\section{Competing interests}

ZYP and FZ report no related interests. JAK has received consulting fees and grant support from Astute Medical and has licensed unrelated technologies through the University of Pittsburgh to Astute Medical.

\section{Authors' contributions}

JAK and ZYP designed the study. FZ and ZYP performed the experiments, and ZYP performed the analysis. JAK and ZYP interpreted the data, and JAK drafted the manuscript. All authors participated in revising the manuscript. All authors read and approved the final manuscript.

\section{Financial support}

This work was funded in part by Astute Medical. The content is solely the responsibility of the authors.

\section{Author details}

${ }^{1}$ Department of Critical Care Medicine, The Center for Critical Care Nephrology, CRISMA (Clinical Research, Investigation, and Systems Modeling of Acute IIIness) Center, University of Pittsburgh School of Medicine, 604 Scaife Hall, 3550 Terrace Street, Pittsburgh, PA 15261, USA. ²Department of Critical Care Medicine, Zhongnan Hospital of Wuhan University School of medicine, Wuhan 630071, China. ${ }^{3}$ Department of Critical Care Medicine, Chinese People's Liberation Army General Hospital, Beijing, China.

Received: 25 February 2016 Accepted: 3 May 2016

Published online: 31 May 2016

\section{References}

1. KDIGO AKIWG (2012) Kidney Disease: Improving Global Outcomes (KDIGO) clinical practice guideline for acute kidney injury. Kidney Inter 2(Suppl 2012):1-138, 1-141

2. Kashani K, Al-Khafaji A, Ardiles T et al (2013) Discovery and validation of cell cycle arrest biomarkers in human acute kidney injury. Crit Care 17:R25

3. Bihorac A, Chawla LS, Shaw AD et al (2014) Validation of cell-cycle arrest biomarkers for acute kidney injury using clinical adjudication. Am J Respir Crit Care Med 189:932-939

4. Yang Q-H, Liu D-W, Long Y et al (2009) Acute renal failure during sepsis: potential role of cell cycle regulation. J Infect 58:459-464

5. Price PM, Safirstein RL, Megyesi J (2009) The cell cycle and acute kidney injury. Kidney Int 76:604-613

6. Boonstra J, Post JA (2004) Molecular events associated with reactive oxygen species and cell cycle progression in mammalian cells. Gene 337:1-13

7. Seo D-W, Li H, Qu C-K et al (2006) Shp-1 mediates the antiproliferative activity of tissue inhibitor of metalloproteinase-2 in human microvascular endothelial cells. J Biol Chem 281:3711-3721

8. Seo D-W, Li H, Guedez L et al (2003) TIMP-2 mediated inhibition of angiogenesis: an MMP-independent mechanism. Cell 114:171-180

9. Kellum JA, Chawla LS (2015) Cell-cycle arrest and acute kidney injury: the light and the dark sides. Nephrol Dial Transplant 31:16-22

10. Kellum JA, Devarajan P (2014) What can we expect from biomarkers for acute kidney injury? Biomark Med 8:1239-1245

11. Zarbock A, Schmidt C, Van Aken $\mathrm{H}$ et al (2015) Effect of remote ischemic preconditioning on kidney injury among high-risk patients undergoing cardiac surgery: a randomized clinical trial. JAMA 313:2133-2141

12. Uchino S, Kellum JA, Bellomo R et al (2005) Acute renal failure in critically ill patients: a multinational, multicenter study. JAMA 294:813-818

13. Hoste EAJ, Bagshaw SM, Bellomo R et al (2015) Epidemiology of acute kidney injury in critically ill patients: the multinational AKI-EPI study. Intensive Care Med 41:1411-1423

14. Zhou F, Peng Z-Y, Bishop JV et al (2013) Effects of fluid resuscitation with $0.9 \%$ saline versus a balanced electrolyte solution on acute kidney injury in a rat model of sepsis*. Crit Care Med 42:e270-e278

15. Bellomo R, Ronco C, Kellum JA et al (2004) Acute renal failure-definition, outcome measures, animal models, fluid therapy and information technology needs: the Second International Consensus Conference of the Acute Dialysis Quality Initiative (ADQI) Group. Crit Care 8:R204-R212

16. DeLong ER, DeLong DM, Clarke-Pearson DL (1988) Comparing the areas under two or more correlated receiver operating characteristic curves: a nonparametric approach. Biometrics 44:837-845

17. Yang Y-L, Liu DD, Hsieh N-K, Chen HI (2008) Endothelin and gelatinases in renal changes following blockade of nitric oxide synthase in hypertensive rats. Chin J Physiol 51:186-195 
18. Douthwaite JA, Johnson TS, Haylor JL et al (1999) Effects of transforming growth factor-beta1 on renal extracellular matrix components and their regulating proteins. J Am Soc Nephrol 10:2109-2119

19. Bach LA, Hale $L$ (2015) Insulin-like growth factors and kidney disease. Am J Kidney Dis 65:327-336

20. Zarbock A, Kellum JA (2015) Remote ischemic preconditioning and protection of the kidney-a novel therapeutic option. Crit Care Med 44:607-16

21. Basu RK, Standage SW, Cvijanovich NZ et al (2011) Identification of candidate serum biomarkers for severe septic shock-associated kidney injury via microarray. Crit Care 15:R273

22. Peng Z-Y, Wang H-Z, Srisawat $\mathrm{N}$ et al (2012) Bactericidal antibiotics temporarily increase inflammation and worsen acute kidney injury in experimental sepsis*. Crit Care Med 40:538-543

23. Kellum JA, Sileanu FE, Murugan R et al (2015) Classifying AKI by urine output versus serum creatinine level. J Am Soc Nephrol 26:2231-2238

24. Kellum JA, Chawla LS, Keener C et al (2016) The effects of alternative resuscitation strategies on acute kidney injury in patients with septic shock. Am J Respir Crit Care Med 193:281-287

\section{Submit your manuscript to a SpringerOpen ${ }^{\circ}$ journal and benefit from:}

- Convenient online submission

- Rigorous peer review

- Immediate publication on acceptance

Open access: articles freely available online

- High visibility within the field

- Retaining the copyright to your article 